\title{
ASYMPTOTIC BEHAVIOR OF A LINEAR DELAY DIFFERENCE EQUATION
}

\author{
R. D. DRIVER, G. LADAS, AND P. N. VLAHOS
}

(Communicated by Kenneth R. Meyer)

ABSTRACT. Consider the linear delay difference equations

$$
x_{n+1}-x_{n}=\sum_{j=1}^{m} a_{j}\left(x_{n-k_{j}}-x_{n-l_{j}}\right), \quad n=0,1,2, \ldots
$$

and

$$
y_{n+1}-y_{n}=\sum_{j=1}^{k} b_{j} y_{n-j}, \quad n=0,1,2, \ldots,
$$

where the coefficients $a_{j}$ and $b_{j}$ are real and $k_{j}$ and $l_{j}$ are nonnegative integers. In this note we describe, in terms of the initial conditions, the asymptotic behavior of solutions of these equations in several cases when the characteristic equation has a dominant real root. Some of the results extend to systems of equations.

Theorem 1 below will apply to a linear delay difference system

$$
\mathbf{x}_{n+1}-\mathbf{x}_{n}=\sum_{j=1}^{m} A_{j}(n)\left(\mathbf{x}_{n-k_{j}}-\mathbf{x}_{n-l_{j}}\right), \quad n=0,1,2, \ldots,
$$

where $m \geq 1$ and for each $j$, the coefficient $A_{j}(n)$ is a square matrix and the delays $k_{j}$ and $l_{j}$ are nonnegative integers. Without loss of generality, assume that $k_{j}>l_{j}$ for each $j$; and let $k=\max _{1 \leq j \leq m} k_{j}$.

Theorems 2,3 , and 4 apply to a special scalar case of (1) and to the linear scalar delay difference equation

$$
y_{n+1}-y_{n}=\sum_{j=1}^{k} b_{j} y_{n-j}, \quad n=0,1,2, \ldots,
$$

where the $b_{j}$ 's are real numbers and $k$ is a positive integer.

An equation like (2) is usually regarded as a difference equation of order $k+1$. This note illustrates the possible advantage of considering (1) and (2) as first-order "delay difference equations" and applying techniques of delay differential equations.

Let $\|\cdot\|$ denote either the norm on the vector space for Eq. (1) or the associated induced norm on square matrices.

Received by the editors August 21, 1990.

1991 Mathematics Subject Classification. Primary 39A11; Secondary 39A05. 
Theorem 1. Let

$$
\sum_{j=1}^{m}\left(k_{j}-l_{j}\right)\left\|A_{j}(n)\right\| \leq \rho<1 \text { for } n=0,1,2, \ldots
$$

Then every solution of (1) tends to a finite limit as $n \rightarrow \infty$.

If each matrix $A_{j}$ is a constant (independent of $n$ ) and the solution of (1) with initial conditions

$$
\mathbf{x}_{n}=\phi_{n} \text { for } n=-k, \ldots, 0,
$$

has a limit (for whatever reason) for every choice of initial conditions, then

$$
\lim _{n \rightarrow \infty} \mathbf{x}_{n}=\left(I+\sum_{j=1}^{m}\left(k_{j}-l_{j}\right) A_{j}\right)^{-1}\left(\phi_{0}+\sum_{j=1}^{m} A_{j} \sum_{i=-k_{j}}^{-l_{j}-1} \phi_{i}\right) .
$$

Proof. Let $\Delta=\max _{-k \leq n \leq-1}\left\|\phi_{n+1}-\phi_{n}\right\|$. Then, by induction on $q=1$, $2, \ldots$,

$$
\left\|\mathbf{x}_{n+1}-\mathbf{x}_{n}\right\| \leq \rho^{q} \Delta \text { for } n \geq q k-k .
$$

Thus $\left\{\mathbf{x}_{n}\right\}$ is a Cauchy sequence and hence converges.

Now let each $A_{j}$ be a constant matrix. Then the quantity

$$
\mathbf{C}_{n} \equiv \mathbf{x}_{n}+\sum_{j=1}^{m} A_{j} \sum_{i=n-k_{j}}^{n-l_{j}-1} \mathbf{x}_{i}
$$

is an invariant, i.e., $\mathbf{C}_{n+1}=\mathbf{C}_{n}$ for $n=0,1, \ldots$. Thus, if $\lim _{n \rightarrow \infty} \mathbf{x}_{n}$ exists,

$$
\left(I+\sum_{j=1}^{m}\left(k_{j}-l_{j}\right) A_{j}\right) \lim _{n \rightarrow \infty} \mathbf{x}_{n}=\lim _{n \rightarrow \infty} \mathbf{C}_{n}=\mathbf{C}_{0}
$$

The matrix on the left side must have full rank since $\mathbf{C}_{0}$ is an arbitrary vector. Hence the matrix is invertible, and Eq. (5) follows.

Remarks. If initial data is chosen randomly, then $\mathbf{C}_{0} \neq 0$ with probability 1 . So, assuming (3) and constant coefficients, Eq. (5) almost always provides a genuine asymptotic representation of the solution of (1).

Note also that with a little more effort the proofs of the theorems in this paper would actually provide estimates of the error.

In the case of constant coefficients and assuming (3), Theorem 1 implies that $\lambda=1$ must be a simple root of the characteristic equation for (1),

$$
\operatorname{det}\left(I(\lambda-1)-\sum_{j=1}^{m} A_{j}\left(\lambda^{-k_{j}}-\lambda^{-l_{j}}\right)\right)=0,
$$

and that all other roots must satisfy $|\lambda|<1$.

Results will be obtained for Eq. (2) in Theorems 2, 3, and 4 by transforming (2) into a scalar special case of (1),

$$
x_{n+1}-x_{n}=\sum_{j=1}^{k} a_{j}\left(x_{n-j}-x_{n}\right) \text { for } n=0,1, \ldots
$$


Lemma. For Eq. (2) assume that the characteristic equation

$$
F(\lambda)=0 \quad \text { where } F(\lambda) \equiv \lambda-1-\sum_{j=1}^{k} b_{j} \lambda^{-j}
$$

has a real root $\lambda_{0}$. Let $\left\{y_{n}\right\}$ be the solution of (2) with initial conditions

$$
y_{n}=\phi_{n} \text { for } n=-k, \ldots, 0,
$$

and define $x_{n}=\lambda_{0}^{-n} y_{n}$ for $n \geq-k$. Then $\left\{x_{n}\right\}$ satisfies Eq. $\left(1^{\prime}\right)$ where $a_{j}=b_{j} \lambda_{0}^{-j-1}$ for $j=0, \ldots, k$.

If $\lim _{n \rightarrow \infty} x_{n}$ exists for every choice of initial values (7), then $\lambda_{0}$ is a simple root of (6), every other root satisfies $|\lambda|<\lambda_{0}$ and

$$
\lim _{n \rightarrow \infty}\left(\lambda_{0}^{-n} y_{n}\right)=\lim _{n \rightarrow \infty} x_{n}=\left(\phi_{0}+\sum_{j=1}^{k} b_{j} \sum_{i=-j}^{-1} \lambda_{0}^{-j-i-1} \phi_{i}\right) /\left(1+\sum_{j=1}^{k} j b_{j} \lambda_{0}^{-j-1}\right) \text {. }
$$

Proof. Introduce $x_{n} \equiv \lambda_{0}^{-n} y_{n}$ for $n \geq-k$. Then $\lambda_{0} x_{n+1}-x_{n}=$ $\sum_{j=1}^{k} b_{j} \lambda_{0}^{-j} x_{n-j}$. Since $\lambda_{0}$ satisfies (6),

$$
{ }_{0} x_{n+1}-{ }_{0} x_{n}=\sum_{j=1}^{k} b_{j} \lambda_{0}^{-j-1}\left(x_{n-j}-x_{n}\right) \text {, }
$$

which is equivalent to Eq. $\left(1^{\prime}\right)$.

Now assume that $\lim _{n \rightarrow \infty} x_{n}$ exists, regardless of the initial conditions. Then, by Theorem $1, \lambda_{0}$ is a simple root of (6), which means $F^{\prime}\left(\lambda_{0}\right)=1+$ $\sum_{j=1}^{k} j b_{j} \lambda_{0}^{-j-1} \neq 0$, and the value of $\lim _{n \rightarrow \infty}\left(\lambda_{0}^{-n} y_{n}\right)=\lim _{n \rightarrow \infty} x_{n}$ is given by (8).

Theorem 2. In Eq. (2) let

$$
\sum_{j=1}^{k}\left|b_{j}\right| \frac{(k+1)^{j+1}}{k^{j}}<1
$$

Then the characteristic equation (6) has exactly one root $\lambda_{0} \in(k /(k+1), \infty)$; and if $\left\{y_{n}\right\}$ is the solution of Eq. (2) with initial conditions (7), then (8) holds. Proof. Define $F(\lambda)$ as in (6) and observe that $F(\infty)=\infty, F(k /(k+1))<0$, and for $\lambda \geq k /(k+1), F^{\prime}(\lambda)>0$. Hence Eq. (6) has exactly one root $\lambda_{0}>k /(k+1)$. Inequality (9) then implies

$$
\sum_{j=1}^{k} j\left|b_{j}\right| \lambda_{0}^{-j-1}<1
$$

Introduce $x_{n} \equiv \lambda_{0}^{-n} y_{n}$ and apply Theorem 1 to the resulting Eq. $\left(1^{\prime}\right)$ to conclude that $\lim _{n \rightarrow \infty} x_{n}$ exists for every solution of $\left(1^{\prime}\right)$. The assertion of the theorem follows from the lemma.

Remarks. Theorem 2 is the discrete analogue of an elementary theorem for a delay differential equation [2]. 
Theorem 2 can be extended to a system of equations if the number of equations is odd. But if the number of equations is even, the characteristic equation may have no real root. Consider, for example, the system

$$
\mathbf{y}_{n+1}-\mathbf{y}_{n}=A \mathbf{y}_{n-1} \quad \text { where } A=\frac{1}{16}\left(\begin{array}{cc}
1 & 1 \\
-1 & 1
\end{array}\right)
$$

If, in addition to the hypotheses of Theorem $2, \sum_{j=1}^{k} b_{j}<0$, then $F(1)>0$ and hence $0<\lambda_{0}<1$. In this case it follows from (8) that every solution of Eq. (2) tends to zero as $n \rightarrow \infty$. Similarly, if $\sum_{j=1}^{k} b_{j}>0$, then $F(1)<0$, $\lambda_{0}>1$, and $y_{n} \rightarrow \infty$ as $n \rightarrow \infty$.

When every $b_{j}$ has the same sign, hypothesis (9) is no longer needed. The results to follow have some overlap with Theorem 2 .

Theorem 3 (cf. deBruijn [1] and Norris [4]). Let $b_{j} \geq 0$ for $j=0, \ldots, k$ with $b_{0}>0$ and consider

$$
y_{n+1}=\sum_{j=0}^{k} b_{j} y_{n-j} \text { for } n=0,1,2, \ldots
$$

- a generalization of (2). Let $\lambda_{0}$ be the unique positive root of the characteristic equation

$$
\lambda-b_{0}-\sum_{j=1}^{k} b_{j} \lambda^{-j}=0 .
$$

Then $\lim _{n \rightarrow \infty} \lambda_{0}^{-n} y_{n}$ exists, and its value is given by (8).

Proof. Case (i). Assume $\sum_{j=0}^{k} b_{j}=1$. Then $\lambda_{0}=1$ and (10) is equivalent to $\left(1^{\prime}\right)$ with $a_{j}=b_{j}$ and $x_{n}=y_{n}$. But Theorem 1 does not apply since $\sum_{j=1}^{k} j b_{j}$ may be greater than 1 .

For $n \geq 0$ define

$$
M_{n} \equiv \max \left\{y_{n-k}, \ldots, y_{n}\right\} \quad \text { and } m_{n} \equiv \min \left\{y_{n-k}, \ldots, y_{n}\right\} \text {. }
$$

Then for any $n \geq 0$ a trivial induction using (10) for $p=0,1,2, \ldots$ shows that

$$
m_{n} \leq m_{n+p} \leq M_{n+p} \leq M_{n} .
$$

Now consider (10) in the form

$$
y_{n+1}=b_{0} y_{n}+\sum_{j=1}^{k} b_{j} y_{n-j} \text { for } n \geq 0
$$

Then it follows by induction on $p$ that

$$
y_{n+p}=b_{0}^{p} y_{n}+\sum_{i=1}^{p} b_{0}^{p-i} \sum_{j=1}^{k} b_{j} y_{n+i-1-j} \text { for } p=1,2, \ldots
$$

Hence for $p \geq 1$

$$
y_{n+p} \leq b_{0}^{p} y_{n}+\sum_{i=1}^{p} b_{0}^{p-i}\left(1-b_{0}\right) M_{n}=M_{n}-b_{0}^{p}\left(M_{n}-y_{n}\right)
$$


and

$$
y_{n+p} \geq b_{0}^{p} y_{n}+\sum_{i=1}^{p} b_{0}^{p-i}\left(1-b_{0}\right) m_{n}=m_{n}+b_{0}^{p}\left(y_{n}-m_{n}\right) .
$$

Considering $1 \leq p \leq k+1$, these yield

$$
M_{n+k+1} \leq M_{n}-b_{0}^{k+1}\left(M_{n}-y_{n}\right) \text { and } m_{n+k+1} \geq m_{n}+b_{0}^{k+1}\left(y_{n}-m_{n}\right) \text {. }
$$

Subtract these last two inequalities to find

$$
M_{n+k+1}-m_{n+k+1} \leq\left(1-b_{0}^{k+1}\right)\left(M_{n}-m_{n}\right) .
$$

It follows that $\left\{M_{n}\right\}$ and $\left\{m_{n}\right\}$ converge to a common limit, $L$. Thus $y_{n} \rightarrow L$ as $n \rightarrow \infty$.

Case (ii). In the general case, Eq. $\left(6^{\prime}\right)$ has a unique positive root $\lambda_{0}$. Define $x_{n}=\lambda_{0}^{-n} y_{n}$. Then $\left\{x_{n}\right\}$ satisfies

$$
x_{n+1}=\sum_{j=0}^{k} b_{j} \lambda_{0}^{-j-1} x_{n-j},
$$

where $\sum_{j=0}^{k} b_{j} \lambda_{0}^{-j-1}=1$. It follows from Case (i) that $\lim _{n \rightarrow \infty}\left(\lambda_{0}^{-n} y_{n}\right)=$ $\lim _{n \rightarrow \infty} x_{n}$ exists.

But Eq. (11) is equivalent to $\left(1^{\prime}\right)$ with $a_{j}=b_{j} \lambda_{0}^{-j-1}$ for $j=1, \ldots, k$. So (8) follows from the lemma.

Remark. Theorem 3 says something about the roots of the polynomial equation, equivalent to $\left(6^{\prime}\right)$,

$$
\lambda^{k+1}-\sum_{j=0}^{k} b_{j} \lambda^{k-j}=0,
$$

where each $b_{j} \geq 0$ and $b_{0}>0$. From the existence of the limit (8), it follows that (12) has a unique positive root $\lambda_{0}$. This root is simple, and for every other root, $|\lambda|<\lambda_{0}$.

Corollary. In Eq. (2) let each $b_{j} \geq 0$. Then Eq. (6) has a unique positive solution $\lambda_{0}$ and (8) holds. Moreover, $\lambda_{0}>1$.

Proof. This is the special case of Theorem 3 when $b_{0}=1$.

Notice that condition (9) in Theorem 2 is irrelevant when each $b_{j} \geq 0$.

Surprisingly, Theorem 3 for equations with positive coefficients is also useful in the study of Eq. (2) with negative coefficients.

Theorem 4. Let $b_{j} \leq 0$ for $j=1, \ldots, k$, and let $\left\{y_{n}\right\}$ be the solution of (2) with initial conditions (7). Assume that at least one $b_{j}<0$, or Eq. (2) becomes trivial. Define $q$ to be the unique positive number such that

$$
F^{\prime}(q)=1+\sum_{j=1}^{k} j b_{j} q^{-j-1}=0,
$$

where $F$ is the characteristic function defined in (6).

(i) If $F(q)<0$, then Eq. (6) has a unique solution $\lambda_{0}$ in $(q, \infty)$ and (8) holds. Moreover $q<\lambda_{0}<1$. 
(ii) If $F(q)=0$, then Eq. (6) has a double root $\lambda_{0}=q<1$, and

$$
\lim _{n \rightarrow \infty}\left(\lambda_{0}^{-n} y_{n}-B n\right)=L \text {, }
$$

where

$$
B=2\left(\phi_{0}+\sum_{j=1}^{k} b_{j} \sum_{i=-j}^{-1} \lambda_{0}^{-j-i-1} \phi_{i}\right) /\left(1+\sum_{j=1}^{k} j^{2}\left|b_{j}\right| \lambda_{0}^{-j-1}\right)
$$

and $L$ is described in the proof below.

(iii) If $F(q)>0$, then $E q$. (6) has no positive roots and $\left\{y_{n}\right\}$ oscillates.

Proof. Note that $F^{\prime}(\lambda)<0$ when $\lambda<q$ and $F^{\prime}(\lambda)>0$ when $\lambda>q$. Thus $F$ attains its minimum in $(0, \infty)$ at $q$.

First assume $F(q) \leq 0$, and let $\lambda_{0}$ be the unique root in $[q, \infty)$ of $F(\lambda)=$ 0 . Since $F(1)>0$, clearly $\lambda_{0} \in[q, 1)$. Once again define

$$
x_{n}=\lambda_{0}^{-n} y_{n} \quad \text { for } n \geq-k ; \quad a_{j}=\lambda_{0}^{-j-1} b_{j} \quad \text { for } j=1, \ldots, k .
$$

Then $x_{n}$ satisfies Eq. $\left(1^{\prime}\right)$.

(i) Let $F(q)<0$. Then $q<\lambda_{0}<1$ and $\sum_{j=1}^{k} j\left|b_{j}\right| \lambda_{0}^{-j-1}<1$, and (8) holds by Theorem 1 .

(ii) (Adapted from Norris [4].) Let $F(q)=0$. Then $\lambda_{0}=q \in(0,1)$ is a double root of the characteristic equation $\left(F^{\prime \prime}(q) \neq 0\right)$, and $\left(1^{\prime}\right)$ holds with

$$
\sum_{j=1}^{k} j\left|a_{j}\right|=\sum_{j=1}^{k} j\left|b_{j}\right| \lambda_{0}^{-j-1}=1 .
$$

As in the proof of Theorem 1,

$$
x_{n}=C_{0}-\sum_{j=1}^{k} a_{j} \sum_{i=n-j}^{n-1} x_{i}
$$

where

$$
C_{0}=x_{0}+\sum_{j=1}^{k} a_{j} \sum_{i=-j}^{-1} x_{i}=\phi_{0}+\sum_{j=1}^{k} b_{j} \sum_{i=-j}^{-1} \lambda_{0}^{-j-i-1} \phi_{i} .
$$

But in this case, unless $C_{0}=0, \lim _{n \rightarrow \infty} x_{n}$ does not exist because of (14).

Define $B=2 C_{0} /\left(1+\sum_{j=1}^{k} j^{2}\left|a_{j}\right|\right)=2 C_{0} /\left(1+\sum_{j=1}^{k} j^{2}\left|b_{j}\right| \lambda_{0}^{-j-1}\right)$, and let $z_{n}=x_{n}-B n$ Then, from (15),

$$
z_{n+1}=\sum_{l=1}^{k}\left|a_{l}\right| \sum_{j=0}^{l-1} z_{n-j}=\sum_{j=0}^{k-1}\left(\sum_{l=j+1}^{k}\left|a_{l}\right|\right) z_{n-j} \text { for } n=0,1, \ldots
$$

This is

$$
z_{n+1}=\sum_{j=0}^{k-1} c_{j} z_{n-j} \text { for } n=0,1, \ldots
$$

where

$$
c_{j} \equiv \sum_{l=j+1}^{k}\left|a_{l}\right| \text { for } j=0, \ldots, k-1 .
$$


Equation (16) is an example of (10). Moreover,

$$
\sum_{j=0}^{k-1} c_{j}=\sum_{j=0}^{k-1} \sum_{l=j+1}^{k}\left|a_{l}\right|=\sum_{l=1}^{k} \sum_{j=0}^{l-1}\left|a_{l}\right|=\sum_{l=1}^{k} l\left|a_{l}\right|=1 .
$$

So, by Theorem 3 ,

$$
\lim _{n \rightarrow \infty}\left(\lambda_{0}^{-n} y_{n}-B n\right)=\lim _{n \rightarrow \infty} z_{n}=\left(z_{0}+\sum_{j=1}^{k-1} c_{j} \sum_{l=-j}^{-1} z_{l}\right) /\left(1+\sum_{j=1}^{k-1} j c_{j}\right),
$$

or, in terms of the original quantities;

$$
\begin{aligned}
\lim _{n \rightarrow \infty}\left(\lambda_{0}^{-n} y_{n}-B n\right)= & 2\left(\phi_{0}+\sum_{i=1-k}^{-1} \sum_{j=1-i}^{k}(j+i)\left|b_{j}\right| \lambda_{0}^{-j-1}\left(\lambda_{0}^{-i} \phi_{i}-B i\right)\right) \\
& \div\left(1+\sum_{j=1}^{k} j^{2}\left|b_{j}\right| \lambda_{0}^{-j-1}\right),
\end{aligned}
$$

where the sum in the numerator is interpreted to be zero if $k=1$.

(iii) Let $F(q)>0$. Then Eq. (6) has no positive roots. The fact that $\left\{y_{n}\right\}$ oscillates in this case follows from an idea of Partheniadis [5] generalized to the present case in [3].

Remark. In the case when all $b_{j} \leq 0$, condition (9) of Theorem 2 implies that $q<k /(k+1)$ and $F(q)<0$ as assumed in Theorem 4(i). However the converse is not true, except in the case of one delay, as the following two examples show.

Example 1. The delay difference equation

$$
y_{n+1}-y_{n}=-\frac{21}{100} y_{n-1}-\frac{1}{100} y_{n-2}
$$

yields $F^{\prime}(1 / 2)=0$ and $F(1 / 2)<0$. So the asymptotic behavior of solutions is described by Theorem 4(i). Theorem 2 is no help since (9) is not satisfied.

Example 2. Consider the delay difference equation with only one delay

$$
y_{n+1}-y_{n}=b y_{n-k} \quad \text { where } b<0 \text {. }
$$

Then (13) becomes $1+k b q^{-k-1}=0$ which yields $q=(k|b|)^{1 /(k+1)}$. This gives

$$
\frac{F(q)}{q}=\frac{k+1}{k}-(k|b|)^{-1 /(k+1)} \text {. }
$$

Thus $F(q)<0$ if and only if $|b|(k+1)^{k+1} / k^{k}<1$. So in the case of one delay and $b<0,(9)$ is the exact condition for Theorem 4(i). Note also, as remarked after Theorem 2 , that in this case $\lambda_{0}<1$. Thus for every solution $y_{n} \rightarrow 0$ as $n \rightarrow \infty$.

If $|b|(k+1)^{k+1} / k^{k}=1$, then $\lambda_{0}=q=k /(k+1)$ is a double root, and (ii) applies.

If $|b|(k+1)^{k+1} / k^{k}>1$ then every solution oscillates.

Example 3. The equation

$$
y_{n+1}-y_{n}=-\frac{3}{25} y_{n-1}-\frac{9}{125} y_{n-2}
$$

is an example of Theorem 4(ii) with $\lambda_{0}=q=3 / 5$. 


\section{REFERENCES}

1. N. G. deBruijn, On some linear functional equations, Publ. Math. Debrecen 1 (1950), 129134. MR 12-106

2. R. D. Driver, D. W. Sasser, and M. L. Slater, The equation $x^{\prime}(t)=a x(t)+b x(t-\tau)$ with "small" delay, Amer. Math. Monthly 80 (1973), 990-995.

3. G. Ladas, Ch. G. Philos, and Y. G. Sficas, Necessary and sufficient conditions for the oscillation of difference equations, Libertas Math. 9 (1989), 121-125.

4. M. J. Norris, unpublished notes on the delay differential equation $x^{\prime}(t)=b x(t-1)$ where $-1 / e \leq b<0$, October 1967.

5. E. C. Partheniadis, Stability and oscillation of neutral delay differential equations with piecewise constant argument, Differential Integral Equations 1 (1988), 459-472.

Department of Mathematics, University of Rhode Island, Kingston, RI 02881 